\title{
Oxidant-induced autophagy and ferritin degradation contribute to epithelial-mesenchymal transition through lysosomal iron
}

This article was published in the following Dove Press journal:

Journal of Inflammation Research

28 March 2017

Number of times this article has been viewed

\author{
Apostolos Sioutas' \\ Linda K Vainikka ${ }^{2}$ \\ Magnus Kentson ${ }^{3}$ \\ Sören Dam-Larsen ${ }^{4}$ \\ Urban Wennerström ${ }^{5}$ \\ Petra Jacobson' \\ Hans Lennart Persson' \\ 'Division of Respiratory Medicine, \\ Department of Medical and Health \\ Sciences, ${ }^{2}$ Division of Experimental \\ Pathology, Department of Clinical \\ and Experimental Medicine, Linköping \\ University, Linköping, ${ }^{3}$ Division \\ of Medicine, Ryhov Hospital, \\ Jönköping, ${ }^{4}$ Division of Medicine, \\ Hospital of Eksjö, Eksjö, ${ }^{5}$ Division \\ of Medicine, Hospital of Västervik, \\ Västervik, Sweden
}

Purpose: Transforming growth factor (TGF)- $\beta 1$ triggers epithelial-mesenchymal transition (EMT) through autophagy, which is partly driven by reactive oxygen species (ROS). The aim of this study was to determine whether leaking lysosomes and enhanced degradation of $\mathrm{H}$-ferritin could be involved in EMT and whether it could be possible to prevent EMT by iron chelation targeting of the lysosome.

Materials and methods: EMT, H-ferritin, and autophagy were evaluated in TGF- $\beta 1$-stimulated A549 human lung epithelial cells cultured in vitro using Western blotting, with the additional morphological assessment of EMT. By using immunofluorescence and flow cytometry, lysosomes and ROS were assessed by acridine orange and 6-carboxy-2',7'-dichlorodihydrofluorescein acetate assays, respectively.

Results: TGF- $\beta 1$-stimulated cells demonstrated a loss of $\mathrm{H}$-ferritin, which was prevented by the antioxidant N-acetyl-L-cysteine (NAC) and inhibitors of lysosomal degradation. TGF- $\beta 1$ stimulation generated ROS and autophagosome formation and led to EMT, which was further promoted by the additional ROS-generating cytokine, tumor necrosis factor- $\alpha$. Lysosomes of TGF- $\beta 1$-stimulated cells were sensitized to oxidants but also completely protected by lysosomal loading with dextran-bound deferoxamine (DFO). Autophagy and EMT were prevented by NAC, DFO, and inhibitors of autophagy and lysosomal degradation.

Conclusion: The findings of this study support the role of enhanced autophagic degradation of $\mathrm{H}$-ferritin as a mechanism for increasing the vulnerability of lysosomes to iron-driven oxidant injury that triggers further autophagy during EMT. This study proposes that lysosomal leakage is a novel pathway of TGF- $\beta 1$-induced EMT that may be prevented by iron-chelating drugs that target the lysosome.

Keywords: A549 cells, pulmonary disease, transforming growth factor, pulmonary fibrosis, tumor necrosis factor, COPD

\section{Introduction}

Inflammation is associated with oxidative injury to pulmonary epithelial cells and is a key event that leads to pulmonary fibrosis, end-stage COPD, and chronic airflow obstruction. ${ }^{1}$ The damaged bronchiolar and alveolar epithelium responds to injury by the proliferation of epithelial cells, including type II pneumocytes, which have a role in pulmonary fibrogenesis via their transformation to fibroblasts through epithelial-mesenchymal transition (EMT). ${ }^{1,2}$ The process of EMT results in epithelial cell loss of their characteristic morphology and phenotype; epithelial cell markers, such as zonula occludens-1 (ZO-1) and E-cadherin, are replaced by mesenchymal markers, including vimentin and $\mathrm{N}$-cadherin. ${ }^{1,3}$ The cytokine,
Correspondence: Hans Lennart Persson Department of Respiratory Medicine and Department of Medical and Health Sciences, Linköping University, Garnisonsvägen, SE-58I 85 Linköping, Sweden

Tel +46 I0 I03 362I

Email lennart.persson@liu.se 
transforming growth factor (TGF)- $\beta$ drives EMT and the development of fibrosis. ${ }^{1,3}$

TGF- $\beta 1$-induced EMT is mediated by an excess of intracellular reactive oxygen species (ROS) ${ }^{3,4}$ TGF- $\beta 1$ inhibits $\gamma$-glutamylcysteine synthetase, the rate-limiting enzyme in the synthesis of the major cellular antioxidant glutathione (GSH). ${ }^{5}$ However, TGF- $\beta 1$ may also induce ROS formation directly. ${ }^{6,7}$ The antioxidant N-acetyl-L-cysteine (NAC) is known to inhibit ROS formation and TGF- $\beta 1$-induced EMT by replenishing intracellular GSH stores. ${ }^{8}$ Tumor necrosis factor- $\alpha$ (TNF- $\alpha$ ) is another ROS and inflammationgenerating cytokine, which is known to augment EMT in the presence of TGF- $\beta 1 .{ }^{9,10}$

Oxidative injury is potentiated by reactive iron $\left(\mathrm{Fe}^{2+}\right)$ through Fenton-type oxidative reactions. ${ }^{11-13}$ Potentially harmful iron is kept in an unreactive state, that is, by being stored in a ferric state $\left(\mathrm{Fe}^{3+}\right)$ in the ferritin molecule. Each ferritin molecule can sequester 4,500 iron atoms per molecule. ${ }^{11-13}$ Light chains (L) and heavy chains (H) form the ferritin molecule, but only the $\mathrm{H}$ chain of ferritin possesses the ferroxidase activity required for iron sequestration..$^{12,13}$ During oxidative stress, ferritin can be damaged by oxidants, resulting in the formation of aggregates, which are recycled through the lysosome. ${ }^{13}$ This process is called "macrophagy" and involves the formation of autophagosomes, which harbor damaged cell material until fusion with lysosomes and formation of autolysosomes. ${ }^{12,13}$ Inside autolysosomes, the cargo is then degraded by lysosomal enzymes working at an acidic $\mathrm{pH} \cdot{ }^{12,13}$ One of the end products is free iron, which, due to the reductive milieu of lysosomes, mainly exists in a redox active state. ${ }^{11-13}$ Following oxidative stress, iron may trigger oxidative damage on lysosomal membranes, resulting in lysosomal leakage. ${ }^{11-13}$ Moderate lysosomal leakage initiates cellular reparative processes, which involve enhanced autophagy. ${ }^{14}$ As a consequence of enhanced autophagy, the cell may become trapped in a self-perpetuating cycle promoting lysosomal damage with further reparative autophagy. Unless the vicious circle of autophagy is broken, it will lead to cell death, inflammation, tissue damage, and fibrosis..$^{12,13}$

Following autophagic uptake of oxidatively damaged ferritin, ${ }^{12-16}$ iron is released from ferritin by lysosomal degradation of the protein. Liberated iron is then transported to the cytosol, forming the cytosolic part of the cell's labile iron pool (LIP). The LIP regulates the synthesis of ferritin and other iron-responsive proteins. ${ }^{12,13}$ Autophagy is now believed to be involved in EMT. ${ }^{17,18}$ In 2009, Zhang et al demonstrated that the LIP and ferritin heavy chain-mediated iron homeostasis followed by increased ROS production were essential for EMT. ${ }^{19}$ In this study, an increase in the LIP in TGF- $\beta 1$-stimulated A549 human type II alveolar epithelial cells in vitro resulted in EMT, which was inhibited in the presence of deferoxamine.

In view of these previously published findings, the present in vitro study included TGF- $\beta 1$-stimulated A549 human type II alveolar epithelial cells based on the experimental approach of Zhang et al. ${ }^{19}$ The aim of the present study was to determine whether leaking lysosomes and enhanced degradation of H-ferritin could be involved in EMT and whether it could be possible to prevent EMT by iron chelation targeting of the lysosome. ${ }^{20,21}$ In addition, the present study included agents known to inhibit autophagy and lysosomal degradation, notably dextran-bound deferoxamine (DFO), which exclusively acts inside lysosomes, to bind iron in a nonreactive state. ${ }^{19}$

\section{Materials and methods Cell cultures and treatments}

Cells of the A549 human type II alveolar epithelial cell line (purchased commercially; ATCC ${ }^{\circledR}$ CCL-185 ${ }^{\mathrm{TM}}$ ) were cultured in Dulbecco's modified Eagle's medium (DMEM) supplemented with $100 \mathrm{IU} / \mathrm{mL}$ penicillin, $100 \mu \mathrm{g} / \mathrm{mL}$ streptomycin (all from GIBCO, Paisley, UK), and 10\% fetal bovine serum (PAA Laboratories, GmBH, Pasching, Austria) at standard culture conditions at $37^{\circ} \mathrm{C}$, in humidified air with $5 \% \mathrm{CO}_{2}$.

Cultured cells were seeded at a density of 1,700 cells $/ \mathrm{cm}^{2}$. After $4 \mathrm{~h}$, attached cells were rinsed with phosphate-buffered saline (PBS), and the culture medium was changed. The following agents were added to the cell cultures at nonlethal concentrations: TGF- $\beta 1,10 \mathrm{ng} / \mathrm{mL}$; 3-methyladenine (3-MA), 0.5-2 mM; NAC, $5 \mathrm{mM}$; bafilomycin A1, $10 \mathrm{nM}$; chloroquine diphosphate salt (CQ), $10 \mu \mathrm{M}$; pepstatin A, $100 \mu \mathrm{M}$; E-64d, $10 \mu \mathrm{M}$ (all from Sigma-Aldrich Inc., St. Louis, MO, USA); lactacystin, $5 \mu \mathrm{M}$ (Calbiochem, San Diego, CA, USA); and TNF- $\alpha, 100$ ng/mL (Bioscience Inc., San Diego, CA, USA). The deferoxamine used was bound to dextran, which meant that DFO was phagocytosed and delivered by endosomes to the lysosomes within a few hours of exposure, acting exclusively inside lysosomes and remaining there until cell death. The DFO concentration used was $0.1 \mathrm{mM}$ in longterm experiments.

Cells were treated with all the above agents for $72 \mathrm{~h}$ before analysis by Western blot or flow cytometry. Some cells were also exposed to a brief 60-min oxidant challenge following a rinse in PBS followed by a stable physiological concentration of $\mathrm{H}_{2} \mathrm{O}_{2}(\sim 120 \mu \mathrm{M})$ at standard culture conditions generated by adding a stock solution of glucose oxidase (GO; Sigma-Aldrich Inc.) to the culture medium. In these 
experiments, $2 \mathrm{mM}$ DFO was added to fresh culture medium $4 \mathrm{~h}$ prior to oxidant challenge.

\section{Western blots}

Total protein was extracted from cells using lysis buffer $(63 \mathrm{mM}$ tris- $\mathrm{HCl}, \mathrm{pH} 6.8,10 \%$ glycerol, $2 \%$ sodium dodecyl sulfate [SDS]), and the protein concentrations were measured by using the Bio-Rad $\mathrm{DC}^{\mathrm{TM}}$ protein assay. The cell lysate samples were run on $16 \%$ SDS-polyacrylamide gel electrophoresis gels and transferred onto nitrocellulose membranes for all antibodies except ferritin, for which polyvinylidene difluoride membrane was used instead. The membranes were blocked in dried skimmed milk powder, incubated with primary antibody overnight at $4^{\circ} \mathrm{C}$, washed with tris-buffered saline (TBS)-Tween, incubated with horseradish peroxidase (HRP)-conjugated secondary antibodies for $1 \mathrm{~h}$ at room temperature, washed again, and finally developed by using Clarity ${ }^{\mathrm{TM}}$ Western extra chemiluminiscent (ECL) substrate. The following primary antibodies were used: ZO-1 (sc-4515; Santa Cruz Biotechnology, Dallas, TX, USA; 1:1,000), E-cadherin (ab1416; Abcam, Cambridge, UK; 1:1,000), N-cadherin (ab76057; Abcam; 1:1,000), vimentin (ab8978; Abcam; 1:2,000), H-ferritin (sc-25617; Santa Cruz Biotechnology; 1:200), and LC3 (NB600-1384; Novus Biologicals, Littleton, CO, USA; 1:1,000). HRP-conjugated glyceraldehyde-3-phosphate dehydrogenase (GAPDH; Novus Biological; 1:50,000) was used as a loading control. The densities of the protein bands were determined, and the optical density of each protein was then adjusted to the density of the corresponding GAPDH band.

\section{Cytofluorometric analysis of lysosomes and ROS}

The metachromatic fluorophore, acridine orange (AO; Gurr, Poole, UK), which is a lysosomotropic base ( $\mathrm{pKa}=10.3)$, was used to assess lysosomes. The "vacuome" is a system of vacuoles that can be stained with neutral red in the living cells; AO red fluorescence was used for the assessment of the acidic vacuome (late endosomes, lysosomes, and autolysosomes). $\mathrm{AO}$ green fluorescence was used for GO-induced lysosomal membrane permeabilization (LMP). AO was trapped in its protonated form as $\mathrm{AOH}^{+}$inside the acidic vacuolar compartment ( $\mathrm{pH}=4.5-5.5)$. Intact lysosomes exhibited red fluorescence due to the high concentration of $\mathrm{AO}$, whereas in cytosol and cell nuclei, a much lower concentration of AO emitted green fluorescence. ${ }^{20,21}$

ROS was assessed by the oxidation of 6-carboxy-2',7'dichlorodihydrofluorescein acetate ( $\left.\mathrm{H}_{2} \mathrm{DCFDA}\right)$ according to the manufacturer's instruction (Molecular Probes, Eugene, OR, USA). A BD LSR flow cytometer (Becton-Dickinson, Mountain View, CA, USA), equipped with a 488-nm exciting argon laser and CellQuest software for data acquisition and analysis, was used.

\section{Statistical analysis}

Results were expressed as the mean \pm standard deviation. Statistical comparisons were made by using analysis of variance. A $p$-value $<0.05$ was considered significant.

\section{Results}

\section{TGF- $\beta$ I exposure resulted in a loss of} $\mathrm{H}$-ferritin through lysosomal degradation

The amount of H-ferritin in cells, which was considered to reflect the total amount of ferritin (H-ferritin and L-ferritin), was assessed by using Western blot. Figure $1 \mathrm{~A}$ and B shows the amount of cellular H-ferritin in TGF- $\beta 1$-stimulated cells following different treatments. Compared with nonstimulated control cells, stimulation with TGF- $\beta 1$ resulted in a loss of $\mathrm{H}$-ferritin as evaluated by Western blot. H-ferritin was minimally detectable in DFO-treated cells. The antioxidant, NAC, almost completely prevented TGF- $\beta 1$-induced loss of H-ferritin. This observation indirectly supported the view that the loss of $\mathrm{H}$-ferritin was partly due to the oxidative modifications of the ferritin molecule. Inhibition of lysosomal degradation, either by CQ or bafilomycin A1, which blocked the formation of autophagolysosomes and inhibited lysosomal degradation by raising intralysosomal $\mathrm{pH}$, or by a mixture of pepstatin $\mathrm{A}$ and E-64d, which specifically blocked lysosomal enzymes, resulted in an accumulation of $\mathrm{H}$-ferritin. The proteasome inhibitor, lactacystin, had no effect on H-ferritin, indicating that the proteasome was not involved.

Collectively, these findings demonstrated that the loss of $\mathrm{H}$-ferritin observed in TGF- $\beta 1$-stimulated cells was due to the oxidative damage to the $\mathrm{H}$-ferritin molecule and resulted in increased degradation of the protein inside lysosomes.

\section{TGF- $\beta$ I-induced oxidative stress, autophagy, and EMT, which were augmented by TNF- $\alpha$}

For the detection of ROS in cells, the probe $\mathrm{H}_{2}$ DCFDA was used, which was sensitive to iron-driven oxidative reactions. TGF- $\beta 1$ treatment of cells resulted in ROS generation (Figure 1C). Compared with GO exposure, which significantly increased ROS, the amount of ROS formed by TGF- $\beta 1$ stimulation was much greater. In contrast, the effect of TNF- $\alpha$ alone on ROS formation was small, whereas the two ROSgenerating cytokines together, TGF- $\beta 1$ and TNF- $\alpha$, resulted 


\section{A}

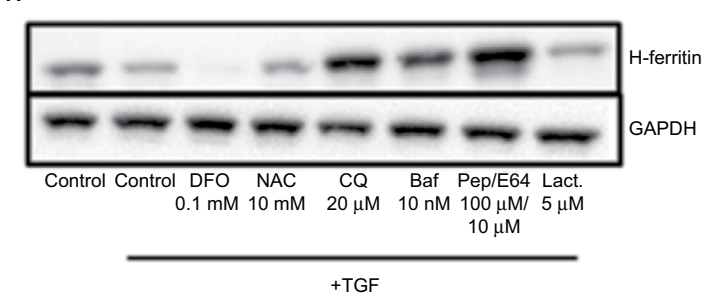

B

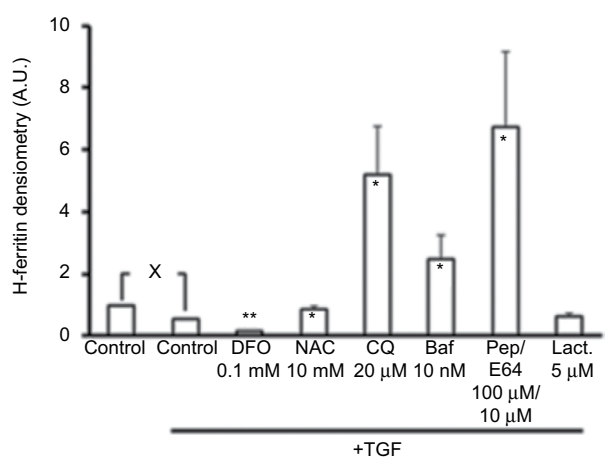

C

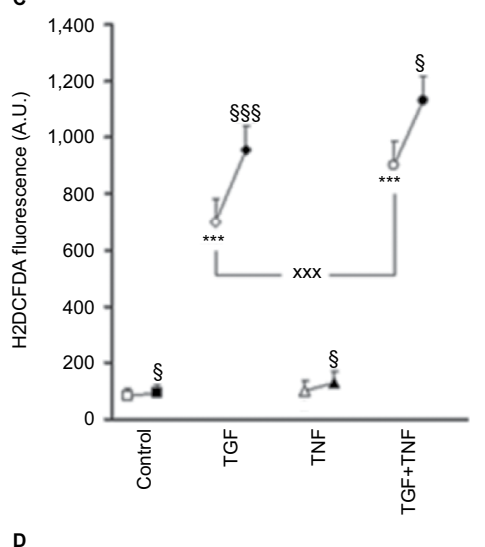

D

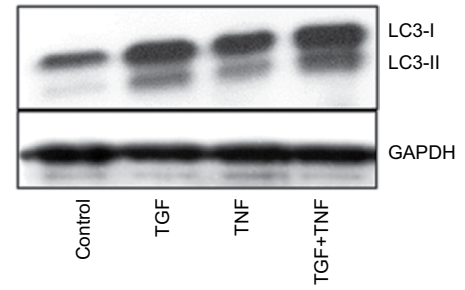

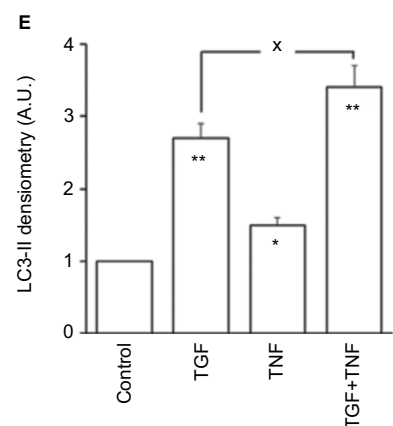

Figure I (A) Representative Western blot and (B) summary of densitometric analysis of $\mathrm{H}$-ferritin in cells following treatment as indicated. A549 cells were exposed (or not) for $72 \mathrm{~h}$ to nonlethal concentrations of TGF- $\beta \mathrm{I}(10 \mathrm{ng} / \mathrm{mL}) \pm$ DFO $(0.1 \mathrm{mM})$, NAC (5 mM), CQ (10 $\mu \mathrm{M})$, baf (I0 nM), a mixture of pepstatin A (I00 $\mu \mathrm{M})$ and E-64d (10 $\mu \mathrm{M})$, or lact $(5 \mu \mathrm{M}) .{ }^{*} p<0.05,{ }^{*} p<0.0 \mathrm{I}$ (vs. TGF- $\beta \mathrm{I}$-exposed control cells); ${ }^{\times} p<0.05$ (vs. control cells). (C) $\mathrm{H}_{2} D C F D A$ fluorescence as a reflection of ROS formation was assessed in cells treated as indicated before (open symbols) and after (symbols in black) GO exposure. A549 cells were exposed (or not) for $72 \mathrm{~h}$ to TGF- $\beta \mathrm{I}$ (I0 ng/mL), TNF- $\alpha$ $(100 \mathrm{ng} / \mathrm{mL})$, or TGF- $\beta \mathrm{I}(10 \mathrm{ng} / \mathrm{mL})+$ TNF- $\alpha(100 \mathrm{ng} / \mathrm{mL})$. Cytofluorometric analysis was performed before and after oxidative stress for $60 \mathrm{~min}$ by a stable physiological concentration of $\mathrm{H}_{2} \mathrm{O}_{2}(\sim \mathrm{I} 20 \mu \mathrm{M})$, generated by adding a stock solution of $\mathrm{GO}$ to the culture medium at standard culture conditions. * $p<0.05$ and $* * * p<0.00 \mathrm{I}$ (vs. non-GOexposed control cells), ${ }^{\S} p<0.05$ and ${ }^{\$ \S} \$ p<0.00$ I (vs. non-GO-exposed cells), ${ }^{x \times x} p<0.00$ I (vs. TGF- $\beta$ I-stimulated cells). (D) Representative Western blot and (E) summary of densitometric analysis of autophagosome formation, reflected by the marker LC3-II in A549 cells following treatments as indicated. ${ }^{*} p<0.05$, ${ }^{* *} p<0.0 \mathrm{I}$ (vs. control); ${ }^{p}<0.05$ (vs. TGF- $\beta$ I). Values are expressed as means \pm I SD, $n=2-5$ (independently performed).

Abbreviations: baf, bafilomycin; CQ, chloroquine diphosphate salt; DFO, dextran-bound deferoxamine; GAPDH, glyceraldehyde-3-phosphate dehydrogenase; GO, glucose oxidase; $\mathrm{H}_{2}$ DCFDA, 6-carboxy-2',7'-dichlorodihydrofluorescein acetate; lact, lactacystin; 3-MA, 3-methyladenine; NAC, N-acetyl-L-cysteine; SD, standard deviation; TGF, transforming growth factor; TNF- $\alpha$, tumor necrosis factor- $\alpha$.

in maximal levels of ROS. The increase in ROS that followed GO exposure was particularly striking in TGF- $\beta 1$-stimulated cultures. This finding supported the view that TGF- $\beta 1$ induced expansion of LIP $^{19}$ and, consequently, the presence of reactive iron that forms ROS in the cytosol by Fenton reactions.

LC3-II was increased following TGF- $\beta 1$ stimulation, indicating an upregulated synthesis of matured autophagosomes (Figure 1D and E). TGF- $\beta 1$ exposure resulted in increased expression of mesenchymal markers, $\mathrm{N}$-cadherin, and vimentin, whereas epithelial markers, ZO-1 and E-cadherin, were downregulated (Figure 2A and B). In support of with these observations, the morphologic appearance of cells shifted from epithelial-like to fibroblast-like (Figure 2C-F).
The combined exposure to TGF- $\beta 1$ and TNF- $\alpha$, both ROS-generating cytokines, resulted in increased autophagy and EMT, shown by EMT markers, ZO-1, E-cadherin, vimentin, and $\mathrm{N}$-cadherin, indicating that oxidative reactions, partly iron-driven, were the mechanisms responsible for both autophagy and EMT.

\section{Inhibition of autophagy attenuated TGF- $\beta$ I-induced ROS formation}

The findings that ROS formation and autophagy were linked and that upregulated autophagy of oxidant-damaged cell material rich in iron promoted the formation of ROS by redox active iron that is liberated from degraded proteins were 
A
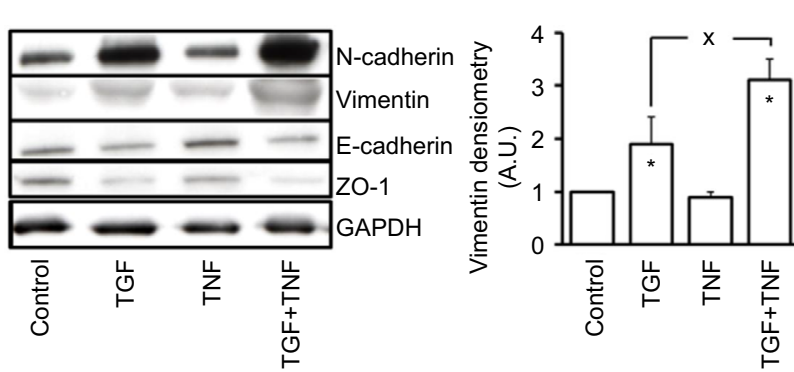

C

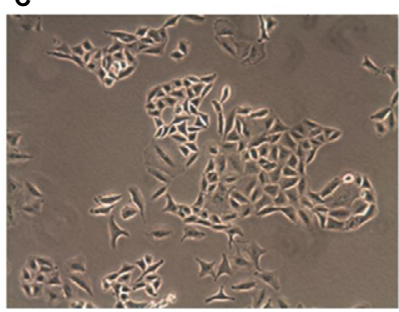

E

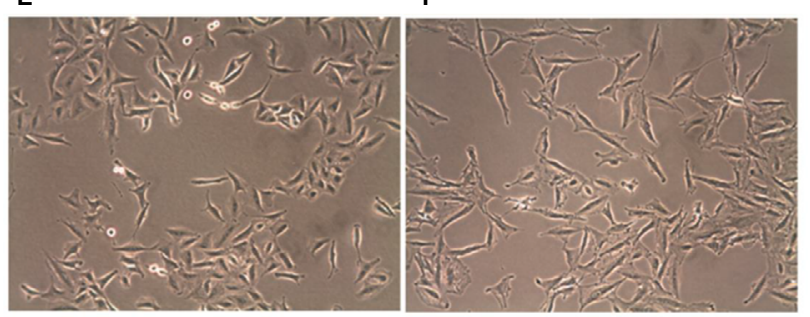

Figure 2 EMT reflected by the expression of cell type-specific markers in cultures treated as indicated. A549 cells were exposed (or not) to nonlethal concentrations of TGF- $\beta$ I (10 ng/mL), TNF- $\alpha(100 \mathrm{ng} / \mathrm{mL})$, or TGF- $\beta$ I $(10 \mathrm{ng} / \mathrm{mL})+$ TNF- $\alpha$ $(100 \mathrm{ng} / \mathrm{mL})$. Cells were treated with the agents for $72 \mathrm{~h}$ before being collected to Western blot. A panel of epithelial markers (ZO-I and E-cadherin) and mesenchymal markers (vimentin and $\mathrm{N}$-cadherin) were tested. (A) Representative Western blot. (B) Summary of densitometric analysis of one representative marker (vimentin). Values are expressed as means \pm I SD, $n=4$ (independently performed). ${ }^{*} p<0.05$ (vs. control), ${ }^{x} p<0.05$ (vs. TGF- $\beta \mathrm{I}$ ). Compared to untreated control cells, corresponding significant changes following the same treatments were demonstrated for ZO-I $(n=3)$, E-cadherin $(n=4)$, and N-cadherin $(n=3)$; results of the densitometric analysis are not shown. Corresponding morphologic appearance of EMT is illustrated by micrographs; (C) control cells, (D) TGF- $\beta$ I-stimulated cells, (E) TNF- $\alpha$-stimulated cells, and (F) TGF- $\beta$ I + TNF- $\alpha$-stimulated cells.

Abbreviations: EMT, epithelial-mesenchymal transition; GAPDH, glyceraldehyde3-phosphate dehydrogenase; SD, standard deviation; TGF, transforming growth factor; TNF- $\alpha$, tumor necrosis factor- $\alpha$; ZO-I, zonula occludens-I.

supported by the results. ${ }^{16}$ However, insufficient autophagy will cause an accumulation of worn-out mitochondria, which are prone to leak excessive amounts of ROS..$^{17,22}$ Both scenarios have been suggested to be involved in EMT and fibrosis. ${ }^{17,18,23,24}$

Thus, in order to clarify the role of autophagy in the formation of ROS, the effect of 3-MA on ROS was investigated, as 3-MA was known to inhibit the formation of autophagosomes by blocking phosphoinositide 3-kinase. In the present study, 3-MA blocked TGF- $\beta 1$-induced formation of autophagosomes as well as ROS in a concentration-dependent manner (Figure 3), which indicated that ROS was mainly generated downstream from autophagosome formation.
A

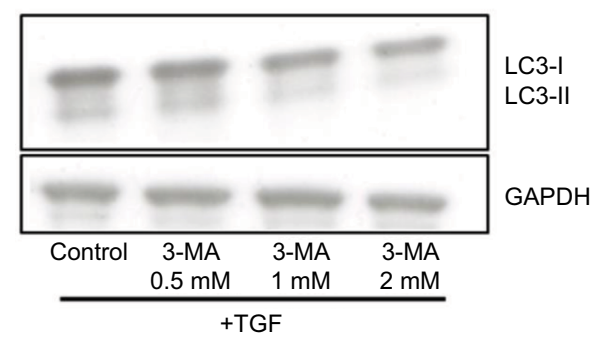

B

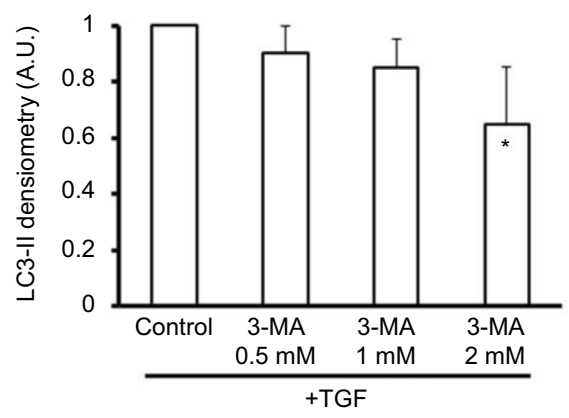

C

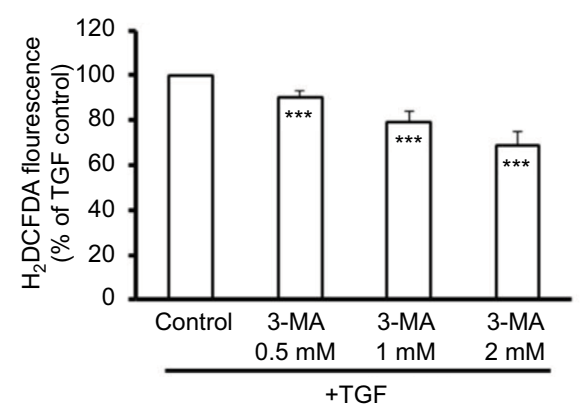

Figure 3 Autophagosome and ROS formation in A549 cells treated with the autophagosome inhibitor 3-MA at nonlethal concentrations. Cells were treated with the agents for $72 \mathrm{~h}$ before being collected to Western blot or analyzed in the flow cytometer. (A) Representative Western blot. (B) Summary of densitometric analysis. Values are expressed as means \pm I SD, $n=2-4$ (independently performed). $*_{p}<0.05$ (vs. TGF- $\beta \mathrm{I}$-exposed cells). (C) $\mathrm{H}_{2}$ DCFDA fluorescence. Values are expressed as means \pm I SD, $\mathrm{n}=5-7$ (independently performed). ${ }^{* * *} p<0.00$ I (vs. TGF- $\beta$ I-exposed cells).

Abbreviations: GAPDH, glyceraldehyde-3-phosphate dehydrogenase; $H_{2}$ DCFDA, 6-carboxy-2',7'-dichlorodihydrofluorescein acetate; 3-MA, 3-methyladenine; ROS, reactive oxygen species; SD, standard deviation; TGF, transforming growth factor; TNF- $\alpha$, tumor necrosis factor- $\alpha$.

\section{Lysosomes of TGF- $\beta$ I-stimulated cells were sensitized to iron-driven oxidant injury}

The acidic vacuome, consisting of late endosomes, lysosomes, and autolysosomes, was evaluated by using the emitted red AO fluorescence. The AO red fluorescence emitted from the acidic vacuome was the sum of two intimately linked processes: autophagy, which expanded the vacuome through the formation of autolysosomes, and lysosomal rupture, which resulted in a loss of intact lysosomes (Figure 4A). Compared with control cells, TGF- $\beta 1$ stimulation increased 


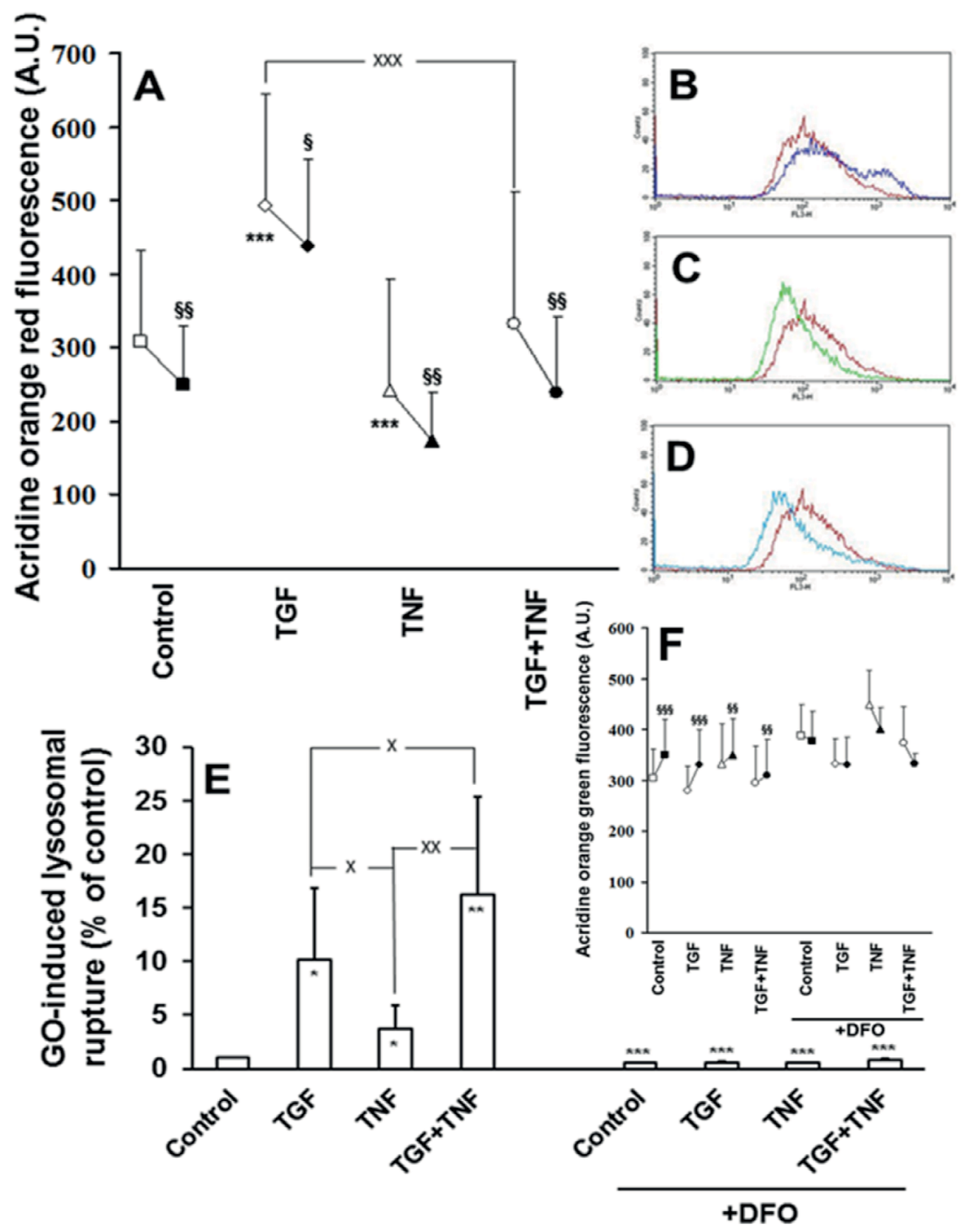

Figure 4 (A) AO induced red fluorescence reflecting the acidic vacuome was assessed following treatments as indicated before (open symbols) and after (symbols in black) GO exposure. A549 cells were exposed (or not) for $72 \mathrm{~h}$ to nonlethal concentrations of TGF- $\beta$ I (I0 ng/mL), TNF- $\alpha$ (I00 ng/mL), or TGF- $\beta$ I (I0 ng/mL) + TNF- $\alpha$ $(100 \mathrm{ng} / \mathrm{mL})$. Cytofluorometric analysis was performed before and after oxidative stress for $60 \mathrm{~min}$ by a stable physiological concentration of $\mathrm{H}_{2} \mathrm{O}_{2}(\sim 120 \mu \mathrm{M})$, generated by adding a stock solution of GO to the culture medium at standard culture conditions. Note the significantly greater loss of AO red fluorescence following GO in TGF$\beta I$-stimulated cells $(p<0.05)$. Values are expressed as means \pm I SD, $n=7$ (independently performed). ${ }^{* * *} p<0.00 \mathrm{I}$ (vs. non-GO-exposed control cells), ${ }^{\$} p<0.05$ and ${ }^{\$ \$} p<0.0 \mathrm{I}$ (vs. non-GO-exposed cells), ${ }^{x \times x} p<0.00$ I (vs. TGF- $\beta$ I-stimulated cells). B-D illustrate the representative flow cytometry curves of non-GO-exposed control cells (red lines; B-D), TGF- $\beta$ I-exposed cells (purple line; B), TNF- $\alpha$-exposed cells (green line; C), and TGF-I $\beta+$ TNF- $\alpha$-exposed cells (blue line; $\mathbf{D}$ ). In separate experiments ( $n=4$; independently performed), GO-induced lysosomal rupture was assessed in the presence of DFO or absence of DFO (E). In these experiments, cells were loaded with DFO (or not) immediately prior to GO exposure ( $2 \mathrm{mM} \mathrm{DFO}$ for $4 \mathrm{~h}$ ). (F) AO-induced green fluorescence reflecting leakage from lysosomes was assessed following treatments as indicated before (open symbols) and after (symbols in black) GO exposure. A549 cells were exposed (or not) as indicated. Cytofluorometric analysis was performed before and after oxidative stress for $60 \mathrm{~min}$ by a stable physiological concentration of $\mathrm{H}_{2} \mathrm{O}_{2}$. Values are expressed as means $\pm \mathrm{I} S \mathrm{SD}, \mathrm{n}=4$ (independently performed). ${ }^{\S \S} \mathrm{p}<0.0 \mathrm{I}$ and ${ }^{s \S} p<0.0$ I (vs. non-GO-exposed cells). ${ }^{*} p<0.05,{ }^{* *} p<0.01,{ }^{\times} p<0.05,{ }^{x} x_{p}<0.01$.

Abbreviations: AO, acridine orange; DFO, dextran-bound deferoxamine; GO, glucose oxidase; SD, standard deviation; TGF, transforming growth factor; TNF- $\alpha$, tumor necrosis factor- $\alpha$.

$\mathrm{AO}$ red fluorescence (Figure $4 \mathrm{~A}$ and $\mathrm{B}$ ), whereas TNF- $\alpha$ stimulation reduced it (Figure 4A and C). Cells stimulated with both TGF- $\beta 1$ and TNF- $\alpha$ exhibited a vacuome similar to control cells (Figure 4A and D), suggesting that the two processes, of autophagy and lysosomal rupture, were in balance.

Independent of prior treatments, $\mathrm{AO}$-induced red fluorescence was decreased by a brief exposure to GO, due to a loss of intact lysosomes (Figure 4A and E). Importantly, GO-dependent decrease of $\mathrm{AO}$ red fluorescence was greater in TGF- $\beta 1$-stimulated cells, than in the cells not stimulated by TGF- $\beta 1$, indicating that TGF- $\beta 1$ stimulation sensitized lysosomes to oxidant injury, possibly through the increased amounts of lysosomal iron (Figure 4E). In order to examine this finding further, lysosomes were loaded with DFO prior to GO exposure, and GO-induced lysosomal rupture was then assessed (Figure 4E). Importantly, DFO prevented lysosomal rupture, indicating that intralysosomal iron-driven oxidative reactions were the major cause of loss of intact lysosomes 
(Figure 4E). Then, leakage of AO, as a reflection of LMP, was assessed as the increase of cytosolic/nuclear AO green fluorescence (Figure 4F). Thus, GO-exposed cells not treated with DFO displayed increased LMP, which was completely prevented in cells with lysosomes loaded with DFO.

\section{Lysosomal iron-chelating blocked TGF- $\beta$ I-induced autophagy}

Iron-driven intralysosomal oxidative reactions, resulting in LMP and lysosomal leakage, were important for the formation of TGF- $\beta 1$-induced autophagosome (Figure $5 \mathrm{~A}$ and B). Furthermore, 3-MA treatment resulted in a significant, but incomplete, decrease in autophagosomes, shown by an increase of LC3-II. CQ and bafilomycin A1, agents blocking the fusion of autophagosomes and lysosomes, caused an accumulation of autophagosomes in cells, demonstrated by a large increase of LC3-II. NAC hampered autophagosome formation similar to 3-MA, whereas DFO suppressed autophagosome formation almost completely. The latter observation indicated that iron-dependent intralysosomal oxidative reactions were a major cause of the upregulation of autophagy observed in TGF- $\beta 1$-stimulated cells.

\section{Lysosomal iron chelating prevented TGF- $\beta$ I-induced EMT}

The effects of CQ and bafilomycin A1 on autophagy were dual as both these agents blocked the fusion of autophagosomes with lysosomes and the activity of lysosomal enzymes by raising the $\mathrm{pH}$ inside lysosomes. These agents were also the most efficient ones to prevent EMT (Figure 5C-E). Prevention of EMT was also achieved by 3-MA, NAC, and DFO treatment, but to a lesser extent. The effects of 3-MA, NAC, and DFO on EMT (Figure 5C-E) were similar to their effects on autophagy (Figure 5A and B).

\section{Discussion}

The findings of this study have shown that TGF- $\beta 1$-stimulated A549 human lung epithelial cells cultured in vitro demonstrated a loss of $\mathrm{H}$-ferritin, which was prevented by the antioxidant NAC and inhibitors of lysosomal degradation. TGF- $\beta 1$
A

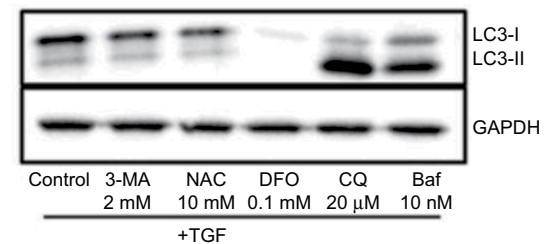

B

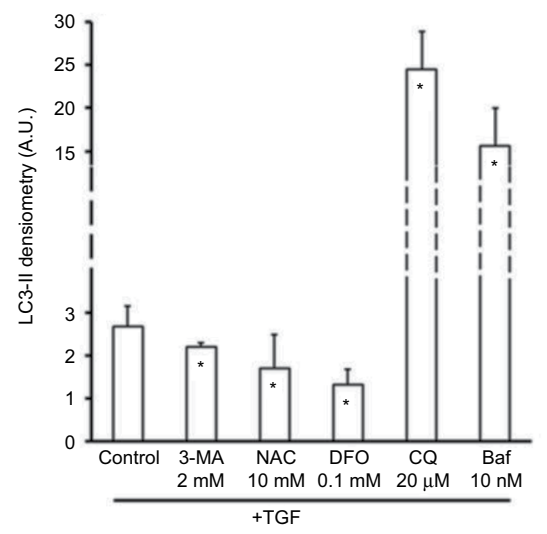

C

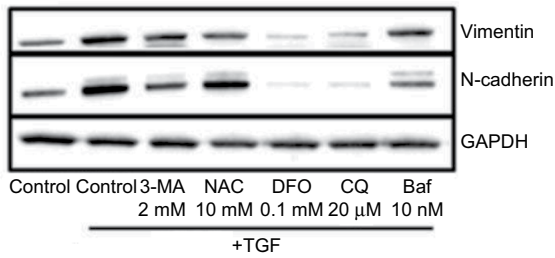

D

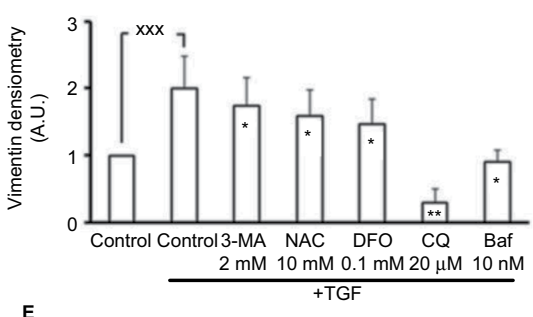

E

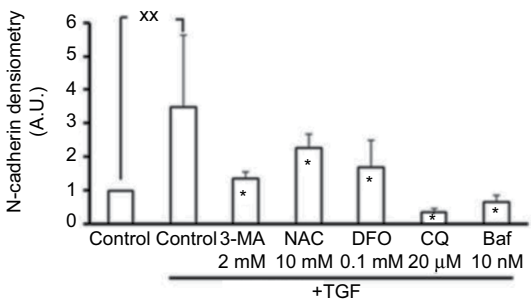

Figure 5 (A) Representative Western blot and (B) summary of densitometric analysis of autophagosome formation in TGF- $\beta$ I-stimulated cells treated as indicated. A549 cells were exposed (or not) for $72 \mathrm{~h}$ to nonlethal concentrations of TGF- $\beta$ I $(10 \mathrm{ng} / \mathrm{mL}) \pm 3-M A(2 \mathrm{mM}), \mathrm{NAC}(5 \mathrm{mM})$, DFO (0.I mM), CQ (I0 $\mu$ M), or Baf (I0 nM). Values are expressed as means \pm I SD, $n=3-7$ (independently performed), ${ }^{*} p<0.05$ (vs. TGF- $\beta$ I-exposed control cells). Control cells were set to I A.U., and corresponding controls (not treated with TGF- $\beta$ I) were all $\leq$ I A.U. (data not shown). (C) Representative Western blots and (D and E) summaries of densitometric analysis of two representative markers of EMT, (D) vimentin and (E) N-cadherin, in A549 cultures treated as indicated. Values are expressed as means \pm I SD, $n=3-4$ (independently performed), * $p<0.05$ (vs. TGF- $\beta$ I-exposed control cells) ${ }^{* *} p<0.0$ I. ${ }^{x x_{p}<0.0 I}$, ${ }^{x \times} x_{p}<0.00$ I. Control cells were set to I A.U., and corresponding controls (not treated with TGF-I $\beta$ ) were all $\leq 1$ A.U. (data not shown).

Abbreviations: Baf, bafilomycin; CQ, chloroquine diphosphate salt; DFO, dextran-bound deferoxamine; EMT, epithelial-mesenchymal transition; GAPDH, glyceraldehyde-3phosphate dehydrogenase; 3-MA, 3-methyladenine; NAC, N-acetyl-L-cysteine; SD, standard deviation; TGF, transforming growth factor; TNF- $\alpha$, tumor necrosis factor- $\alpha$. 
stimulation caused ROS and autophagosome formation and led to EMT, which was further increased by the additional ROSgenerating cytokine, TNF- $\alpha$. Lysosomes of TGF- $\beta 1$-stimulated cells were sensitized to oxidants but also completely protected by lysosomal loading with DFO. Autophagy and EMT were prevented by the antioxidant NAC, DFO, and inhibitors of autophagy and lysosomal degradation.

EMT is now believed to be the mechanism that leads to endstage lung fibrosis, which is found in a variety of inflammatory interstitial lung diseases ${ }^{25}$ and in COPD. ${ }^{1,2}$ Enhanced autophagy and an accumulation of autophagosomes, demonstrated by different autophagy-related proteins, have been reported in lung biopsies from patients with COPD. ${ }^{24}$ Cells from fibrotic tissues exhibit signs of TGF stimulation and enhanced autophagy. ${ }^{24}$ However, published observations are conflicting, as some studies suggest that autophagy is upregulated by TGF, ${ }^{23,24}$ whereas some other studies do not. ${ }^{17,18}$ In the present study, there were striking similarities in the responses to the treatments applied to lung epithelial cells cultured in vitro (Figure 5A-E), and the findings supported previous reports that TGF- $\beta 1$-induced autophagy and EMT are closely linked. ${ }^{23,24}$ In the present study, the expression of the autophagy marker, LC3-II, increased almost threefold following TGF- $\beta 1$ stimulation (Figures $1 \mathrm{D}$ and $\mathrm{E}$ and $5 \mathrm{~A}$ and $\mathrm{B}$ ) and of the EMT markers, vimentin and $\mathrm{N}$-cadherin, increased by two- or threefold (Figures 2 and $5 \mathrm{C}-\mathrm{E})$. The expression of the epithelial marker E-cadherin demonstrated a similar reduction in response to TGF- $\beta 1$ (results not shown). However, in the present study, the inhibition of autophagy by $3-\mathrm{MA}$ (at $2 \mathrm{mM}$ ) reduced the expression of LC3-II by $20 \%-40 \%$ in TGF- $\beta 1$-stimulated cultures (Figures 3 and $5 \mathrm{~A}$ and $\mathrm{B}$ ) and also reduced the expression of vimentin and N-cadherin by $10 \%$ and $60 \%$, respectively (Figure $5 \mathrm{C}-\mathrm{E}$ ). As a result of an enhanced autophagy and upregulated degradation of ferritin, the cellular level of $\mathrm{H}$-ferritin decreased almost $50 \%$ in TGF- $\beta 1$-stimulated cultures (Figure 1). Inhibition of autophagy by 3-MA maintained the cellular level of $\mathrm{H}$-ferritin in TGF- $\beta 1$-stimulated cultures (data not shown). These findings indicate a relationship between autophagy and EMT. These findings are also supported by a recent study by Ghavami et al using different cell lines. ${ }^{26}$

The findings of this study provide evidence to support the existence of a novel pathway to EMT through lysosomal leakage, which involves increased lysosomal degradation of ferritin, which, in the present study, was assessed by H-ferritin as a reflection of total ferritin, $\mathrm{H}$-ferritin and L-ferritin. In addition, the findings of this study also support the possibility of secondary sensitization of lysosomes to oxidant challenge by iron-driven intralysosomal oxidative reactions. These findings are also supported by a previous study using a similar experimental model. ${ }^{19}$ Zhang et al demonstrated that free iron is a link to EMT and observed an increase of iron in TGF- $\beta 1$ stimulated A549 cells, which was explained by a reduced synthesis of $\mathrm{H}$-ferritin. ${ }^{19}$ The latter observation was suggested to be the result of a repressed translation of H-ferritin mRNA. ${ }^{19}$ The present study provides an alternative mechanism to the reduced level of $\mathrm{H}$-ferritin observed in TGF- $\beta 1$-stimulated A549 cells, specifically a loss of protein by degradation in lysosomes secondary to upregulated autophagy of oxidatively damaged ferritin. In support of the findings by Zhang et al, ${ }^{19}$ the present study showed that deferoxamine bound to dextran (DFO), which exclusively targeted the lysosome, worked efficiently to prevent TGF- $\beta 1$-induced EMT.

The present study is the first to address the role of irondriven oxidative lysosomal damage for TGF- $\beta 1$-induced autophagy and EMT. The findings are supported by previous investigators, ${ }^{19}$ but the present study also showed that ROS was increased in TGF- $\beta 1$-stimulated A549 cells and that cells stimulated with two ROS-generating cytokines, TGF- $\beta 1$ and TNF- $\alpha$, responded with a synergistic increase in ROS, which was associated with enhanced autophagy and EMT. Given the previously observed TGF- $\beta 1$-induced increase in iron in the same experimental model, ${ }^{19}$ the findings of the present study support the view that redox active iron is released from leaky lysosomes. In previous studies, TGF- $\beta 1$ triggered oxidantinduced autophagy, but the extent varied between studies. ${ }^{4,27}$ This finding may be explained by the different amounts of oxidants and antioxidants used in previous studies and by the activity of the antioxidative systems available. In the present model, most of the autophagic activity was prevented by DFO, targeting the lysosome, which indicated that the autophagy observed in TGF- $\beta 1$-stimulated cells was mainly dependent on iron-driven oxidative reactions taking place in the lysosome. Furthermore, TGF- $\beta 1$ stimulation sensitized lysosomes to oxidant challenge, and oxidant-induced damage leading to leakage was fully prevented by DFO, again suggesting a role for lysosomal rupture leading to TGF- $\beta 1$-induced autophagy. Detailed analysis of the mechanisms behind TGF- $\beta 1$ induced lysosomal-mediated pulmonary pathology due to fibrosis may be investigated in future by applying inhibitors of the autophagic process at different levels, as this in vitro study showed upregulation of the degradation of $\mathrm{H}$-ferritin, which was secondary to autophagy enhanced by ROS.

In the present study, treatment of TGF- $\beta 1$-stimulated A549 cells with DFO resulted in less H-ferritin, a finding that is in line with previous studies and is explained by two different mechanisms acting through either intact or nonintact lysosomes. ${ }^{11,12,16,20,21}$ Thus, DFO will permanently bind iron inside lysosomes, and once it is released from the degraded ferritin 
molecule, the passage of iron over normal intact lysosomal membranes is impaired; and as part of the LIP, ferritin synthesis is hindered. ${ }^{11,12,16,20,21} \mathrm{~A}$ less well-known mechanism for lysosomal iron to exit lysosomes, and trigger ferritin synthesis, is through nonintact leaking lysosomal membranes following membrane damage. ${ }^{28,29}$ If lysosomal membrane damage involves iron-driven oxidative reactions, DFO will maintain iron in a nonreactive state, and leakage of iron and the resulting upregulation of ferritin synthesis will be prevented. ${ }^{11,12,16,20,21}$

The findings of the present study, combined with previously reported studies ${ }^{20,21}$ and with more recent studies by other groups, ${ }^{30,31}$ provide indirect evidence that iron-driven ROS, generated inside lysosomes, are involved in lysosomal damage. Without access to a suitable sensor for the detection of lysosomal ROS, the importance of ROS generation has been based so far on the detection of reduced levels of ROS, and lysosomal damage, in the presence of iron chelators that specifically target the lysosomal compartment. ${ }^{20,21,30-32}$ A recently published study has shown that mucolipin 1, a member of the transient receptor potential channel family, is a ROS sensor localized on the lysosomal membrane and indicates that lysosome-specific ROS sensors may soon be developed. ${ }^{33}$

Therefore, from the present study, it is possible to conclude that TGF- $\beta 1$ stimulation can sensitize lysosomes to oxidative injury. Surprisingly, cell cultures exposed to TGF- $\beta 1$ for $72 \mathrm{~h}$ did not demonstrate pronounced lysosomal disruption, as was the case for TNF- $\alpha$-stimulated cultures. The response of the acidic vacuome of TGF- $\beta 1$ - and TNF- $\alpha$ stimulated cells contrasts with the large amounts of ROS produced in TGF- $\beta 1$-stimulated cells and the small amount of ROS produced in cells stimulated by TNF- $\alpha$. These findings can only be explained by the presence of protective mechanisms in TGF- $\beta 1$-stimulated cells that counteract the effect of ROS and iron on lysosomal membranes. Recently published studies have shown that areas of damage to plasma membranes can be sealed through the process of lysosomal exocytosis. ${ }^{34}$ Patches of membranes of intact lysosomes can be incorporated into the plasma membrane, thereby strengthening areas weakened by previous damage. We propose that autophagosomes may act in the same way for leaky lysosomes moderating, but not completely inhibiting, TGF- $\beta 1$-induced lysosomal leakage (Figure 6).

\section{Conclusion}

The findings of the present study support the role of enhanced autophagic degradation of $\mathrm{H}$-ferritin as a mechanism for increasing the vulnerability of lysosomes to iron-driven oxidant injury that triggers further autophagy during EMT. This

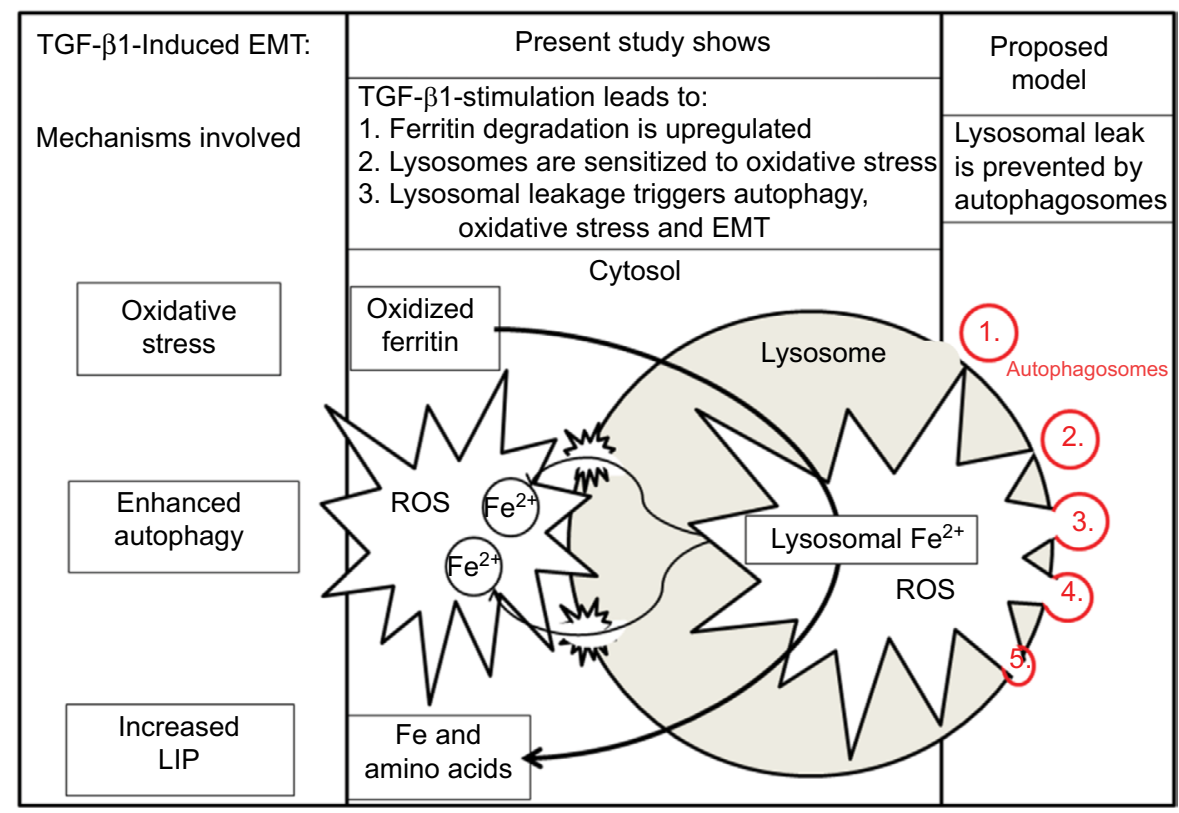

Figure 6 A schematic figure illustrating the intimate link between TGF- $\beta$ I-induced degradation of ferritin and lysosomal leakage. An extensive body of previous studies has demonstrated that TGF- $\beta$ I stimulation triggers EMT and oxidative stress. Similarly, a role for an enhanced autophagy, partly driven by oxidative cellular damage, is established in TGF- $\beta$ I-induced EMT. Recently, Zhang et al demonstrated that TGF- $\beta$ I-stimulation results in increased amounts of iron in the LIP, leading to ROS generation and EMT. ${ }^{19}$ The present study shows for the first time that increased degradation of ferritin inside lysosomes, which is upregulated by an enhanced autophagy, sensitizes the lysosomes to ROS, resulting in iron-driven oxidative injury upon lysosomal membranes. The resulting leakage of iron and harmful lysosomal content into the cytosol triggers further oxidative reactions, autophagy and EMT. Compared to TGF- $\beta$ I-stimulated cultures, TNF- $\alpha$-stimulated cultures display much less ROS and, yet, a significant loss of lysosomes. These observations cannot be explained unless protective mechanisms, working in TGF- $\beta$ I-stimulated cells, counteract lysosomal leakage. Considering the recent finding that protection of damaged plasma membranes is afforded through lysosomal exocytosis, a similar protection of leaky lysosomes by the fusion with a great number of autophagosomes (I-3) is proposed, which thereby may seal the damaged parts of the lysosomal membranes $(4,5)$.

Abbreviations: EMT, epithelial-mesenchymal transition; LIP, labile iron pool; ROS, reactive oxygen species; TGF, transforming growth factor; TNF- $\alpha$, tumor necrosis factor- $\alpha$. 
study proposed that lysosomal leakage is a novel pathway of TGF- $\beta 1$-induced EMT that may be prevented by ironchelating drugs that target the lysosome.

\section{Acknowledgments}

The authors would like to thank Linnea LaFleur for technical assistance. This study was financially supported by grants to H.L.P. from the County Council of Östergötland and the Medical Research Council of Southeast Sweden. The abstract of this paper was presented at the American Thoracic Society 2016 as a poster presentation with interim findings. The abstract was published in American Journal of Respiratory and Critical Care Medicine 193; 2016: A5879: http:/www.atsjournals.org/ doi/book/10.1164/ajrccm-conference.2016.C71

\section{Author contributions}

All the authors contributed toward the study design, implementation, and data analysis; were involved in drafting the manuscript; and read and approved the final draft.

\section{Disclosure}

The authors report no conflicts of interest in this work.

\section{References}

1. Willis BC, Borok Z. TGF-beta-induced EMT: mechanisms and implications for fibrotic lung disease. Am J Physiol Lung Cell Mol Physiol. 2007;293(3):L525-L534.

2. Nowrin K, Sohal SS, Peterson G, Patel R, Walters EH. Epithelialmesenchymal transition as a fundamental underlying pathogenic process in COPD airways: fibrosis, remodeling and cancer. Expert Rev Respir Med. 2014;8(5):547-559.

3. Cannito S, Novo E, di Bonzo LV, Busletta C, Colombatto S, Parola M. Epithelial-mesenchymal transition: from molecular mechanisms, redox regulation to implications in human health and disease. Antioxid Redox Signal. 2010;12(12):1383-1430.

4. Liu R-M, Gaston Pravia KA. Oxidative stress and glutathione in TGF- $\beta$ mediated fibrogenesis. Free Radic Biol Med. 2010;48(1):1-15.

5. Arsalane K, Dubois CM, Muanza T, et al. Transforming growth factor-beta1 is a potent inhibitor of glutathione synthesis in the lung epithelial cell line A549: transcriptional effect on the GSH rate-limiting enzyme gammaglutamylcysteine synthetase. Am J Respir Cell Mol Biol. 1997;17(5):599-607.

6. Hu T, Ramachandrarao SP, Siva S, et al. Reactive oxygen species production via NADPH oxidase mediates TGF- $\beta$-induced cytoskeletal alterations in endothelial cells. Am J Physiol Renal Physiol. 2005;289(4):F816-F825.

7. Perez LM, Milkiewicz P, Ahmed-Choudhury J, et al. Oxidative stress induces actin-cytoskeletal and tight-junctional alterations in hepatocytes by a $\mathrm{Ca}^{2+}$-dependent, PKC-mediated mechanism: protective effect of PKA. Free Radic Biol Med. 2006;40(11):2005-2017.

8. Felton VM, Borok Z, Willis BC. N-acetylcysteine inhibits alveolar epithelial-mesenchymal transition. Am J Physiol Lung Cell Mol Physiol. 2009;297(5):L805-L812.

9. Yamauchi Y, Kohyama T, Takizawa H, et al. Tumor necrosis factor-alpha enhances both epithelial-mesenchymal transition and cell contraction induced in A549 human alveolar epithelial cells by transforming growth factor-beta1. Exp Lung Res. 2010;36(1):12-24.

10. Kawata M, Koinuma D, Ogami T, et al. TGF- $\beta$-induced epithelialmesenchymal transition of A549 lung adenocarcinoma cells is enhanced by pro-inflammatory cytokines derived from RAW 264.7 macrophage cells. J Biochem. 2012;151(2):205-216.
11. Brunk UT, Zhang H, Roberg K, Öllinger K. Lethal hydrogen peroxide toxicity involves lysosomal iron-catalyzed reactions with membrane damage. Redox Rep. 1995;1(4):267-277.

12. Kurz T, Eaton JW, Brunk UT. The role of lysosomes in iron metabolism and recycling. Int J Biochem Cell Biol. 2011;43(12):1686-1697.

13. Linder MC. Mobilization of stored iron in mammals: a review. Nutrients. 2013;5(10):4022-4050.

14. Persson HL, Kurz T, Eaton JW, Brunk UT. Radiation-induced cell death: importance of lysosomal destabilization. Biochem J. 2005;389(Pt 3): $877-884$.

15. Kiffin R, Bandyopadhyay U, Cuervo AM. Oxidative stress and autophagy. Antioxid Redox Signal. 2006;8(1-2):152-162.

16. Persson HL. Radiation-induced lysosomal iron reactivity: implications for radioprotective therapy. IUBMB Life. 2006;58(7):395-401.

17. Patel AS, Lin L, Geyer A, et al. Autophagy in idiopathic pulmonary fibrosis. PLoS One. 2012;7(7):e41394.

18. Araya J, Kojima J, Takasaka N, et al. Insufficient autophagy in idiopathic pulmonary fibrosis. Am J Physiol Lung Cell Mol Physiol. 2013; 304(1):L56-L69.

19. Zhang KH, Tian HY, Gao X, et al. Ferritin heavy chain-mediated iron homeostasis and subsequent increased reactive oxygen species production are essential for epithelial-mesenchymal transition. Cancer Res. 2009;69(13):5340-5348.

20. Persson HL, Yu Z, Tirosh O, Eaton JW, Brunk UT. Prevention of oxidantinduced cell death by lysosomotropic iron chelators. Free Radic Biol Med. 2003;34(10):1295-1305.

21. Yu Z, Persson HL, Eaton JW, Brunk UT. Intralysosomal iron: a major determinant of oxidant-induced cell death. Free Radic Biol Med. 2003;34(10):1243-1252.

22. Zhang H, Kong X, Kang J, et al. Oxidative stress induces parallel autophagy and mitochondria dysfunction in human glioma U251 cells. Toxicol Sci. 2009;110(2):376-388.

23. Gajewska M, Gajkowska B, Motyl T. Apoptosis and autophagy induced by TGF- $\beta 1$ in bovine mammary epithelial BME-UV1 cells. $J$ Physiol Pharmacol. 2005;56 (Suppl 3):143-157.

24. Kiyono K, Suzuki HI, Matsuyama H, et al. Autophagy is activated by TGF- $\beta$ and potentiates TGF- $\beta$-mediated growth inhibition in human hepatocellular carcinoma cells. Cancer Res. 2009;69(23): 8844-8852.

25. Hügle T. Immunology of fibrotic lung disease: managing infections whilst preventing autoimmunity? J Inflamm Res. 2011;4:21-27.

26. Ghavami S, Cunnington RH, Gupta S, et al. Autophagy is a regulator of TGF- $\beta 1$-induced fibrogenesis in primary human atrial myofibroblasts. Cell Death Dis. 2015;6:e1696.

27. Xu YF, Yang SY, Huang JY, Ruan SW, Zheng Z, Lin JM. TGF- $\beta 1$ induced autophagy and promoted apoptosis in renal tubular epithelial cells. Int J Mol Med. 2012;29(5):781-790.

28. Kurz T, Leake A, Von Zglinicki T, Brunk UT. Relocalized redox-active lysosomal iron is an important mediator of oxidative-stress-induced DNA damage. Biochem J. 2004;378(Pt 3):1039-1045.

29. Ghosh M, Carlsson F, Laskar A, Yuan XM, Li W. Lysosomal membrane permeabilization causes oxidative stress and ferritin induction in macrophages. FEBS Lett. 2011;585(4): 623-629.

30. Visalli G, Bertuccio MP, Iannazzo D, Piperno A, Pistone A, Di Pietro A. Toxicological assessment of multi-walled carbon nanotubes on A549 human lung epithelial cells. Toxicol In Vitro. 2015;29(2): 352-262.

31. Tang Y, Shen Y, Huang L, et al. In vitro cytotoxicity of gold nanorods in A549 cells. Environ Toxicol Pharmacol. 2015;39(2):871-878.

32. Kurz T, Gustafsson B, Brunk UT. Intralysosomal iron chelation protects against oxidative stress-induced cellular damage. FEBS J. 2006;273(13):3106-3117.

33. Zhang X, Cheng X, Yu L, et al. MCOLN1 is a ROS sensor in lysosomes that regulates autophagy. Nat Commun. 2016;7:12109.

34. Eding CB, Domert J, Wäster P, Jerhammar F, Rosdahl I, Öllinger K. Melanoma growth and progression after ultraviolet $\mathrm{A}$ irradiation: impact of lysosomal exocytosis and cathepsin proteases. Acta Derm Venereol. 2015;95(7):792-797. 
The Journal of Inflammation Research is an international, peer-reviewed open access journal that welcomes laboratory and clinical findings on the molecular basis, cell biology and pharmacology of inflammation including original research, reviews, symposium reports, hypothesis formation and commentaries on: acute/chronic inflammation; mediators of

Submit your manuscript here: https://www.dovepress.com/journal-of-inflammation-research-journa

inflammation; cellular processes; molecular mechanisms; pharmacology and novel anti-inflammatory drugs; clinical conditions involving inflammation. The manuscript management system is completely online and includes a very quick and fair peer-review system. Visit http://www.dove press.com/testimonials.php to read real quotes from published authors. 